\title{
To the justification of the calculation of the heat conduction process in the dough piece
}

\author{
Evgeniy Semenov ${ }^{1}$, Yury Sidorenko ${ }^{1}$, Igor Nikitin $^{1}{ }^{*}$, Nikolay Bulychev $^{2,3}$, \\ Svetlana Dimitrieva ${ }^{2,1}$ \\ ${ }^{1}$ K.G. Razumovsky Moscow State University of technologies and management \\ (The First Cossack University), St. Zemlyanoy Val, 73, Moscow, Russia \\ ${ }^{2}$ P.N. Lebedev Physical Institute of Russian Academy of Sciences, \\ 119991, Leninsky pr-t, 53, Moscow, Russia \\ ${ }^{3}$ Moscow Aviation Institute (National Research University), \\ 125993, Volokolamskoe shosse, 4, Moscow, Russia
}

\begin{abstract}
The main process that ensures the production of bread as a marketable product is the external thermal energy supply during baking. This process is accompanied by a complex physical phenomenon moisture exchange between the dough-bread with the external environment and the steam-air environment of the baking chamber, as well as internal heat and mass transfer in the heated dough piece. At the same time, biochemical processes associated with the transformation of starch and proteins. Taking into account that the main factor realizing the efficiency and quality indicators of baked bread is heat exchange in the dough piece, in this article, taking into account the peculiarities of the dynamics of the heat transfer phenomenon, a quantitative analysis of the course of this process is carried out. In this work, in contrast to the results obtained by other researchers in the field of calculating the processes of heat treatment of food products, a quantitative analysis of the process is proposed based on a new methodological approach to the problem of physical and mathematical substantiation of the heat transfer process in a dough piece. That can be effectively used in the simulation of innovative samples of baking equipment.
\end{abstract}

\section{Introduction}

It is well known that bakery products are the main source of providing the human body with nutrients and dietary fibers necessary for normal life. It is also known the technology of obtaining high-quality bread by heat treatment of dough pieces, established over many millennia of experience of human civilization. At the same time, both before and now, the problem remains, on the one hand, providing an intense thermal effect on the dough in order to obtain a high-quality product according to a certain technological regime, on the other hand, the implementation of this process with a minimum consumption of energy resources. To resolve this contradiction, it is required, having data on the dynamics of the temperature field during the heat treatment of the dough piece, to organize optimal control

\footnotetext{
* Corresponding author: nikitinia@mgutm.ru
} 
of the baking process. This can be done only on the basis of the rheological constants of the dough and theoretical analysis of the actual course of the heat treatment process. The work [1-5] is devoted to the studying the problems of the baking process of bakery products, where methods of obtaining a high-quality bread product and the issues of resource-saving technologies for controlling the technological process of baking bread are considered in detail [3]. Theoretical and experimental studies are devoted to the search for ways to improve this process from the standpoint of developing energy-saving technologies $[1,2,4,5]$.

Below, in the work, in contrast to other scientific studies, based on the factor of kinematic features of heat propagation noted in real conditions, the substantiation of physical and mathematical modeling of the process of heat treatment of the dough piece and a numerical analysis of the process of heat transfer in this substance are carried out.

\section{Material and methods}

Formulation of the problem. From the point of view of the classical theory, the phenomenon of thermal conductivity is interpreted as a molecular diffusion transfer of thermal energy with an infinite velocity of propagation of a disturbance from a source, as a result of which the temperature of the medium is also assumed to change instantly at each point. However, since this is not observed in real conditions, the concepts of perturbed and unperturbed, separated by the so-called temperature front $x=\rho(t)$ (Fig. 1), of the temperature fields of these phases are introduced [6].

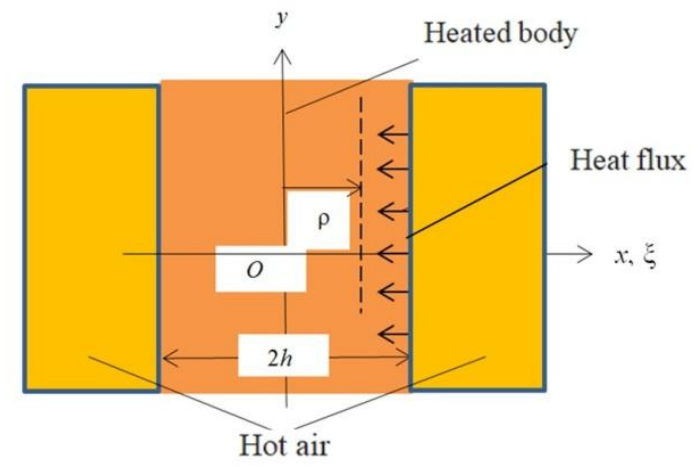

Fig. 1. Scheme for calculating the heat transfer process in the dough piece

In this case, for a heated body at the front, the following conditions are fulfilled: 1) the temperature $\mathrm{T}$ is equal to the temperature of the unperturbed region; 2) the temperature $\partial T / \partial x$ at the front is assumed to be zero, which entails a smooth continuous distribution of both the temperature and the heat flux in the disturbed region in the entire investigated body.

The problem is investigated: for given geometrical and physical and mechanical parameters of the heat transfer process, to obtain, taking into account the factor of the presence of a temperature front, a quantitative estimate of the dynamics of heat propagation in the investigated body in terms of time.

It is believed that the heat transfer in the body is realized in a symmetric manner with respect to the $y$-axis, and, in addition, for the selected computational model, with satisfactory accuracy, all assumptions on the physical and mechanical properties of the object under study are usually made when analyzing the heat transfer process in a continuous medium. 
Since some other baked goods such as bread are characterized by an elongated (along the $y$-axis - Fig. 1) shape, it is believed that the body itself approaches a rectangular parallelepiped in shape, and the initial temperature in the body is taken to be the same throughout the volume.

Then, by virtue of the accepted symmetry condition, the half-space $0 \leq x \leq h$ is taken as an approximate geometric model of the dough piece, to which heat is supplied from the region $x>h$ (Fig. 1).

For the purpose of quantitative analysis of the problem of heat conduction in the investigated body, the equation [7] is used

$$
\partial T / \partial t=a^{2} \partial^{2} T / \partial x^{2}
$$

where $t$ is time, $a^{2}=\lambda /(c \gamma)$ is the coefficient of thermal diffusivity, $\lambda$ is the coefficient of thermal conductivity, $c$ is the heat capacity, $\gamma$ is the density of the body.

In this case, if $T_{0}$ is the initial temperature in the body, then the solution of equation (1) is matched with the initial condition

$$
T(x, 0)=T_{0}=\text { const, } 0 \leq x \leq h .
$$

Then, since when heating in the middle (center) of the body $x=0$, the temperature has a minimum value, the boundary condition along the $y$ axis has the form

$$
\partial T(0, t) / \partial x=0,0<t<\infty
$$

In turn, the condition for the balance of heat fluxes at the boundary between the body and air is written as

$$
\left.\left[\partial T / \partial x-\left(T_{1}-T\right) \alpha / \lambda\right]\right|_{x=h}=0
$$

where $T_{1}$ is the temperature of the environment surrounding the body, $\alpha$ is the heat transfer coefficient, $\lambda$ is the heat conductivity coefficient [7].

The solution of the problem. In the future, in order to simplify the study carried out, dimensionless quantities are introduced according to the dependencies

$$
\left(T-T_{0}\right) / \Delta T=u, x / h=\xi, a^{2} t / h^{2}=\tau, \alpha h / \lambda=\sigma, \Delta T=T_{1}-T_{0}>0,
$$

where $u$ and $\xi$ are dimensionless quantities, $\tau$ is an analogue of the Fourier number, $\sigma$ is an analogue of the Biot number.

In this case, problem (1) - (4) takes the form

$$
\begin{gathered}
\partial u / \partial \tau=\partial^{2} u / \partial \xi^{2}, \\
u(\xi, 0)=0, \\
\partial u(0, t) / \partial \xi=0, \\
\partial u(1, t) / \partial \xi+\sigma u(1, t)=\sigma .
\end{gathered}
$$

Using the so-called averaging method, when studying the problem taking into account the presence of a temperature front, solve it in two phases, replacing the left side of equation (6) with its averaged value over the interval $(\rho, 1)$, namely, introduce into consideration the function [7] 


$$
2 \varphi(\tau)=\frac{1}{1-\rho} \int_{\rho}^{1} \frac{\partial u}{\partial \tau} d \xi
$$

where $\rho$ is the dimensionless coordinate of the temperature front.

Then, instead of equation (6), an approximate relation is used

$$
\partial^{2} u / \partial \xi^{2}=2 \varphi(\tau)
$$

satisfying, according to (7) - (9), the conditions

$$
\begin{gathered}
u(\rho, \tau)=0, \\
{[\partial u(\xi, \tau) / \partial \xi]_{\xi=\rho}=0,} \\
{[\partial u(\xi, \tau) / \partial \xi+\sigma u(\xi, \tau)]_{\xi=1}=\sigma,}
\end{gathered}
$$

and, in addition, equality (10).

Below, if we restrict ourselves to the study of the problem in the first phase of the process, then the solution of the formulated boundary value problem formulated by relations (10) - (14) has the form

$$
\tau=(1-\rho)^{2} / 12+(1-\rho) /(3 \sigma)-2 \ln [1+\sigma(1-\rho) / 2] /(3 \sigma)^{2} .
$$

Solving (15) (numerically) with respect to $\rho$ as an implicit function, the temperature front $\rho=\rho(\tau)$ is determined.

The duration $\tau_{1}$ of the first phase is found by substituting $\rho=0$ in (15)

$$
\tau_{1}=1 / 12+1 /(3 \sigma)-2 \ln (1+\sigma / 2) /\left(3 \sigma^{2}\right) .
$$

Numerical experiment. The initial data were taken (conditionally): the transverse size of the dough piece $1=45 \mathrm{~mm}$; density $\gamma=1180 \mathrm{~kg} / \mathrm{M}^{3}$; thermal conductivity coefficient $\lambda=0,15 \mathrm{~W} /(\mathrm{M} \cdot \mathrm{K})$; heat capacity $c=1700 \mathrm{~J} /(\mathrm{kg} \mathrm{K})$; thermal diffusivity coefficient $a=\lambda /(c \rho)=7,48 \times 10^{-8} \mathrm{~m}^{2} / \mathrm{s}$; heat transfer coefficient $\alpha=20 \mathrm{~W} /\left(\mathrm{M}^{2} / \mathrm{s}\right)$; the initial temperature of the workpiece body is $T_{0}=293 \mathrm{~K}$; air temperature $T_{1}=493 \mathrm{~K}$.

The approximate value of the Fourier criterion (at $t=60 \mathrm{~min}$ ) Fo $=a t / l^{2}=0.108$; criterion $\mathrm{Bi}=\alpha l / \lambda=6.67$.

\section{Results and discussion}

The numerical analysis of the heat transfer process in the dough piece, carried out on the basis of the selected parameter values, taking into account dependencies (5), in dimensional form, is reflected in the graphs in Fig. 2.

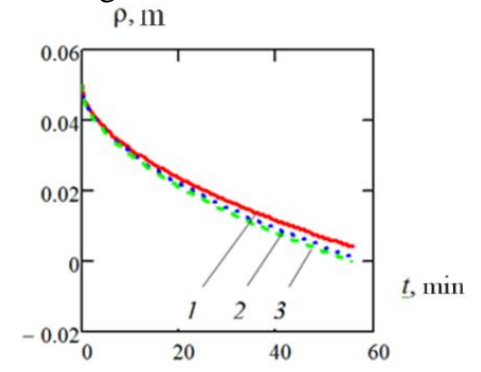

Fig. 2. Dependences of the coordinate $\rho$ of the temperature front on time $\mathrm{t}(1-\mathrm{Bi}=5 ; 2-\mathrm{Bi}=10$; $3-\mathrm{Bi}=15)$. 
The duration of the first phase $\tau_{1}$ (time for the temperature front to reach the center of the workpiece) is calculated according to (16), if in formula (15) is assumed to be $\rho=0$. Analysis of the behavior of curves calculated with the help of the root software product of the Mathcad environment Fig. 2 indicates, in a natural way, an exponentially slower approach of the temperature front to the center of the workpiece over time. In addition, a slowdown of the front is noted ( although insignificant) when the number of Bi grows (for example, due to the thickness of the workpiece): in Fig. 2 curves 2,3 are below curve 1.

\section{Conclusions}

In this work, within the framework of the formulation of the classical linear problem of thermal conductivity in a body simulating a dough piece in the form of a rectangular layer, physical and mathematical modeling and a numerical experiment were carried out, formulated as a boundary problem with boundary conditions of the third kind. On the basis of the transfer of thermal energy from the heat source to the body, carried out taking into account the kinematic features, in a criterial form, temperature data consistent with the physical meaning were obtained. In terms of the implementation of energy-saving technologies, the computational algorithm proposed in the article can be effectively used.

\section{References}

1. V.A. Bryazun, V.I. Maklyukov, M.F. Adnodvortsev, The baking process of baked goods. Theoretical foundations of food technology, P. 814-838, (M.: «KolosS», 2009)

2. V.A. Bryazun, V.I. Maklyukov, M.F. Adnodvortsev, The baking process of baked goods. Theoretical foundations of food technology, P. 838-867, (M.: «KolosS», 2009)

3. E.A. Nemova St. Petersburg. XLIV scientific and educational conference of ITMO University. St. Petersburg, 192 (2015)

4. V.I. Maklyukov Confectionery and bakery production. 5-6, 46, (2016)

5. V.I. Maklyukov Bread products. 2, 59, (2019)

6. A.M. Brazhnikov, V.A. Karpychev, A.I. Peleev Analytical methods for studying the processes of heat treatment of meat products, (Moscow: Food industry, 1974)

7. A.V. Lykov, Heat conduction theory, (M.: Vysshaya shkola, 1967) 